\title{
BMJ Open Enhancing community participation for stroke survivors with cognitive impairment: study protocol for a randomised controlled trial in Taiwan
}

\author{
Feng-Hang Chang (D) , ${ }^{1,2}$ Valeria Chiu, ${ }^{1,3}$ Pengsheng Ni, ${ }^{4}$ Yen-Nung Lin, ${ }^{1,5}$ \\ Jiunn-Horng Kang, ${ }^{2,6}$ Tsan-Hon Liou, ${ }^{2,7}$ Lu Lu, ${ }^{8}$ Der-Sheng Han, ${ }^{9,10}$ \\ Elizabeth R Skidmore ${ }^{11,12}$
}

To cite: Chang F-H, Chiu V, $\mathrm{Ni}$ P, et al. Enhancing community participation for stroke survivors with cognitive impairment: study protocol for a randomised controlled trial in Taiwan. BMJ Open 2020;10:e040241. doi:10.1136/ bmjopen-2020-040241

- Prepublication history and additional material for this paper are available online. To view these files, please visit the journal online (http://dx.doi. org/10.1136/bmjopen-2020040241).

Received 09 May 2020 Revised 31 0ctober 2020 Accepted 02 November 2020

Check for updates

(c) Author(s) (or their employer(s)) 2020. Re-use permitted under CC BY-NC. No commercial re-use. See rights and permissions. Published by BMJ.

For numbered affiliations see end of article.

Correspondence to Dr Feng-Hang Chang; fhchang@tmu.edu.tw

\section{ABSTRACT}

Introduction Stroke can lead to life-long disability and constitutes a huge financial burden on the family and society. Stroke survivors with cognitive impairment often experience considerable challenges in the process of recovery and returning to society. Interventions that effectively help individuals resume essential daily activities and return to active participation in their communities are lacking. This study examines the efficacy of a newly-developed intervention programme, the Optimising Participation after Stroke through Strategytraining (OPASS) programme, for improving community participation among stroke survivors with cognitive impairment.

Methods and analysis A single-blind, parallel-group randomised controlled trial with allocation concealment and assessor blinding will be implemented to assess the efficacy of the OPASS programme. An expected 210 adults with cognitive impairment following stroke will be randomly assigned to either the experimental intervention (OPASS) group or the attention control group. In addition to their usual rehabilitation, both groups will receive $45 \mathrm{~min}$ sessions, twice weekly for a total of 12-15 sessions. The primary outcome is change in participation performance, which will be measured using the participation measurethree domains, four dimensions scale. Additional measures include the Activity Measure for Post-Acute Care generic outpatient short forms, Montreal Cognitive Assessment, Stroop Test, Trail Making Test and General Self-Efficacy Scale. These scales will be administered at baseline, post-intervention, 3-month follow-up, 6-month follow-up and 12-month follow-up. Their results will be analysed using multiple linear regression models and mixed-effects regression models. Further assessment of feasibility and acceptability of the intervention will be conducted through structured interviews with participants, caregivers and therapists. These interviews will be transcribed and thematically analysed.

Ethics and dissemination Ethics approval was obtained from the Ethics Committee of Taipei Medical University (approval number: N201804055). The findings will be disseminated through presentations at scientific conferences and through publication in peer-reviewed journals.

Trial registration number NCT03792061; pre-results.
Strengths and limitations of this study

- This study will be the first parallel randomised controlled trial (RCT) to examine the efficacy of strategy training to enhance community participation in stroke survivors with cognitive impairment in an Asian country.

- Both quantitative and qualitative data will be collected at multiple time points.

- The study results could help advance stroke rehabilitation practice and benefit stroke survivors in terms of their health, independence and transition to the community.

- Challenges in participant recruitment and retention are expected given the long-term nature of this study.

- This study is a single-blind RCT in which participants and interventionists will be aware of group assignment, but outcome assessors and data analysts will be blinded to avoid investigator bias.

\section{INTRODUCTION}

Stroke is a leading cause of acquired disability globally. ${ }^{1}$ According to WHO, 15 million people have stroke worldwide annually, of whom 5 million become permanently disabled. ${ }^{2}$ In Taiwan, approximately 230 people have a stroke every day, ${ }^{3}$ contributing to an approximate prevalence of 19.3 per 1000 people. ${ }^{4}$ Even mild stroke can lead to cognitive impairment. ${ }^{5}$ The prevalence of poststroke cognitive impairment ranges from $20 \%$ to $80 \%{ }^{6}$ depending on how precisely cognitive function is measured. Cognitive impairment includes deficits in the domains of attention, executive function, visuospatial ability, memory and language. ${ }^{7}$ Poststroke cognitive impairment can progress to dementia and considerable long-term disability ${ }^{8}$ resulting in poor quality of life. ${ }^{7}$ Cognitive impairment affects survivors and their family's health and well-being. ${ }^{8}$ Loss of productivity and informal 
caregiving costs also lead to tremendous indirect costs and financial burden to society. ${ }^{9}$ Effective rehabilitation programmes that improve community participation, minimise disability and reduce social burden are urgently required.

Participation in the community is a major rehabilitation goal for stroke survivors. ${ }^{10}$ The International Classification of Functioning, Disability and Health defines participation as 'involvement in life situations'. ${ }^{11}$ This definition has been further explicated as 'active involvement in activities that are intrinsically social and occur in a societally-defined context' (Chang and Coster, ${ }^{12}$ p1792). 'Participation restriction'11 denotes changes, limitations or abnormalities that an individual experiences as engaging in life situations, including home management, productivity, social life and community. ${ }^{10} 12$ Stroke survivors can experience a wide range of participation restrictions, such as difficulty in finding a job or social isolation. ${ }^{13}{ }^{14}$ Many survivors cease their activities performed prior to stroke and limit their time outside the home. ${ }^{14}$ These changes can lead to deteriorating physical and mental health. ${ }^{15-17}$

Despite the importance of participation for stroke survivors, no intervention to date has demonstrated promising efficacy for enhancing participation outcomes among people with cognitive impairment following stroke. Community-based exercise programmes have shown promise for improving stroke survivors' community participation through improved physical function (eg, community ambulation). ${ }^{18-20}$ However, these programmes do not address the challenges associated with cognitive impairment, and stroke survivors with cognitive impairment are typically excluded from such programmes. For interventions designed to address cognitive impairment (eg, neurocognitive functional training), cognitive functions is occasionally improved, but limited effects are observed for participation. ${ }^{21}$ Therefore, interventions that effectively minimise participation restrictions of individuals with cognitive impairments are essential.

Strategy training is a top-down approach designed to enhance cognitive function through the practice of meaningful activities by survivors in their everyday life. ${ }^{22}$ The purpose of strategy training is to guide individuals to generate problem-solving skills to address challenges that they face in daily activities. ${ }^{23}$ Through strategy training, individuals learn to develop their own strategies to manage challenges and apply these strategies to other similar problems they encounter in daily life. ${ }^{24}$ Strategy training has been applied in various clinical populations, including individuals with stroke and brain injury, and its benefits for enhancing executive cognitive function ${ }^{25} 26$ as well as functional independence ${ }^{27}$ have been revealed. Nevertheless, it is unclear whether strategy training has similar effects on participation, the ultimate rehabilitation goal.

To address this research gap, our research team developed the Optimising Participation after Stroke through Strategy-training (OPASS) programme, a participation-focused programme that uses strategy training principles. The feasibility of this intervention programme has been tested in community-dwelling people with stroke and brain injury in Taiwan, ${ }^{28}$ which showed that participation-focused strategy training is feasible and acceptable for adults after stroke and brain injury in Taiwan. Although preliminary, positive changes were observed in the overall scores on the participation measure-three domains, four dimensions (PM-3D4D) (Cohen's $d=0.46-1.25)$ and in performance of their self-identified goals (Cohen's $d=1.82$ ). On the basis of findings of this feasibility study, we designed the present randomised controlled trial (RCT). The purpose of this trial is to assess the efficacy of the OPASS intervention programme for enhancing participation among community-dwelling people with cognitive impairment after stroke. The following hypotheses will be tested in this project:

1. Participants in the OPASS group will demonstrate significantly higher improvement in participation performance than participants receiving attention control.

2. The improvement in participation will be correlated with improvements in applied cognition, performance of daily activities, executive function and self-efficacy.

3 . The OPASS programme will continue to have significant effects on patients' participation at the 3-month, 6-month and 12-month follow-up compared with that of attention control participants.

\section{METHODS AND ANALYSIS \\ Study design}

This study is a multicentre, parallel-group RCT with allocation concealment and assessor blinding. The study is conducted in the rehabilitation departments of six hospitals (including two medical centres, three regional hospitals and one district hospital) in northern Taiwan. Data collection was initiated on 13 January 2019, and is expected to continue until 31 December 2023.

Recruited participants will be randomly assigned to the treatment group (ie, OPASS) and the attention control group at a 1:1 ratio at each site. Outcome assessments will be administered at baseline and at 3, 6 and 12 months after the intervention. A flow diagram of the study design is illustrated in figure 1. The study protocol is reported according to the Standard Protocol Items: Recommendations for Intervention Trials 2013 (SPIRIT). A SPIRIT checklist and the schedule of enrolment, interventions and assessments are provided in the online supplemental file 1 and figure 2 .

\section{Participants}

Participants are recruited from the rehabilitation outpatient wards of the collaborating hospitals. The inclusion criteria are as follows: (1) being adults aged 20 years and older, (2) having a confirmed diagnosis of stroke, (3) being able to understand Mandarin, (4) having cognitive impairment (a score of 3 or more on the 14-item version 


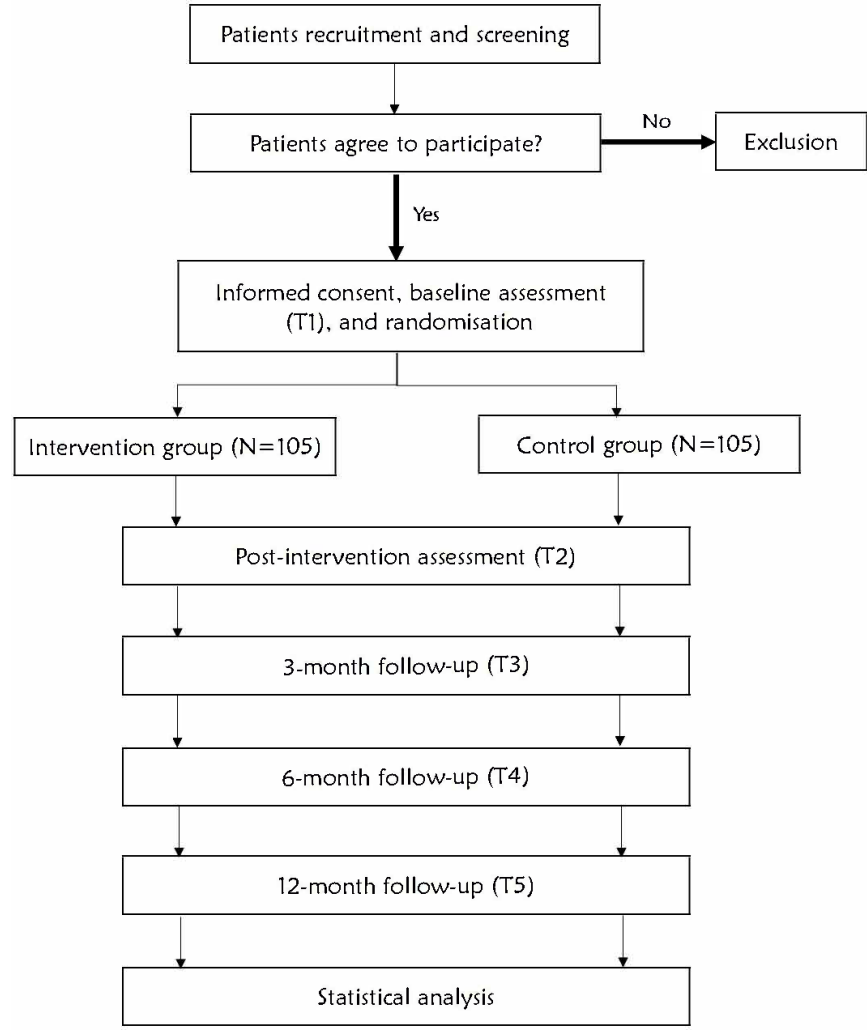

Figure 1 Standard Protocol Items Recommendations for Intervention Trials 2013 flow diagram of the study design.

of Executive Interview ${ }^{29}$ and (5) being able to provide informed consent.

Exclusion criteria are as follows: (1) having severe aphasia and (2) having a prestroke diagnosis of dementia, current major depressive disorder, substance use or other psychiatric disorders that may impede them from continually participating in the study.

\section{Recruitment and screening}

Based on inclusion criteria, our trained research staff coordinates with clinicians at our collaborating sites to screen for eligible participants through medical chart review and personal interviews. The research staff also posts advertisements for this study (eg, posters and flyers) at the collaborating sites. After initial screening, eligible participants receive oral and written information regarding the trial from research staff, and informed consent is obtained from all participants who agree to participate in the study.

\section{Randomisation and allocation concealment}

After baseline assessment, the eligible participants at each recruitment site will be randomly assigned to either the intervention or control group in equal numbers. The random allocation sequence has been calculated using SPSS V.19 (IBM) by an independent researcher who is not involved in the recruitment, evaluation and intervention of participants. Before intervention commencement, the research staff will inform eligible participants of their intervention allocation by telephone.

\begin{tabular}{|c|c|c|c|c|c|c|c|}
\hline \multicolumn{8}{|c|}{ STUDY PERIOD } \\
\hline \multirow[b]{2}{*}{ TIMEPOINT } & \multirow{2}{*}{$\frac{\text { Pre-intervention }}{-t_{1}}$} & \multirow{2}{*}{$\frac{\text { Baseline }}{0}$} & \multicolumn{2}{|c|}{ Intervention } & \multicolumn{3}{|c|}{ Follow-up } \\
\hline & & & $t_{1}$ & $\mathrm{t}_{2}$ & $t_{3}$ & $t_{4}$ & $t_{5}$ \\
\hline \multicolumn{8}{|l|}{ ENROLMENT: } \\
\hline Eligibility screen & $x$ & & & & & & \\
\hline Informed consent & $x$ & & & & & & \\
\hline Allocation & & $x$ & & & & & \\
\hline \multicolumn{8}{|l|}{ INTERVENTIONS: } \\
\hline \multicolumn{8}{|l|}{$\begin{array}{l}\text { Intervention group } \\
\text { (OPASS program) }\end{array}$} \\
\hline $\begin{array}{r}\text { Control group } \\
\text { (non-active intervention) } \\
\end{array}$ & & & $\bullet$ & $\rightarrow$ & & & \\
\hline \multicolumn{8}{|l|}{ ASSESSMENTS: } \\
\hline \multicolumn{8}{|l|}{ Primary outcome: } \\
\hline $\begin{array}{r}\text { Participation Measure-3 } \\
\text { Domains, } 4 \text { Dimensions (PM- } \\
\text { 3D4D }\end{array}$ & & $x$ & & $x$ & $\mathrm{x}$ & $x$ & $x$ \\
\hline \multicolumn{8}{|l|}{ Secondary outcomes: } \\
\hline $\begin{array}{l}\text { Activity Measure for Post-Acute } \\
\text { Care (AM-PAC) }\end{array}$ & & $x$ & & $x$ & $x$ & $x$ & $x$ \\
\hline $\begin{array}{r}\text { Montreal Cognitive Assessment } \\
\text { (MoCA) }\end{array}$ & & $x$ & & $x$ & $\mathrm{x}$ & $\mathrm{x}$ & $x$ \\
\hline Color-naming Stroop Test & & $x$ & & $\mathrm{x}$ & $\mathrm{x}$ & $x$ & $x$ \\
\hline $\begin{array}{l}\text { Trail-Making Test } \\
\text { (TMT A and B) }\end{array}$ & & $\mathrm{x}$ & & $x$ & $\mathrm{x}$ & $\mathrm{x}$ & $x$ \\
\hline $\begin{array}{l}\text { General Self-Efficacy Scale } \\
\text { (GSES) }\end{array}$ & & $x$ & & $x$ & $x$ & $x$ & $x$ \\
\hline \multicolumn{8}{|l|}{ Other variables } \\
\hline Demographic questionnaire & & $x$ & & & & & \\
\hline $\begin{array}{l}\text { National Institutes of Health } \\
\text { Stroke Scale (NIHSS) }\end{array}$ & & $x$ & & $x$ & $x$ & $x$ & $x$ \\
\hline Modified Rankin Scale (MRS) & & $x$ & & $x$ & $x$ & $x$ & $x$ \\
\hline $\begin{array}{r}\text { Health-related Quality of life } \\
\text { (EQ-5D) }\end{array}$ & & $x$ & & $x$ & $\mathrm{x}$ & $\mathrm{x}$ & $x$ \\
\hline $\begin{array}{l}\text { HEAL Positive Outlook } \\
\text { questionnaire }\end{array}$ & & $x$ & & $x$ & $x$ & $x$ & $x$ \\
\hline $\begin{array}{r}\text { Pittsburgh Rehabilitation } \\
\text { Participation Scale }\end{array}$ & & & 。 & $\longrightarrow$ & & & \\
\hline $\begin{array}{l}\text { Strategy Training Fidelity } \\
\text { Checklist }\end{array}$ & & & $\bullet$ & $\longrightarrow$ & & & \\
\hline Qualitative interviews & & & & $x$ & & & \\
\hline
\end{tabular}

Figure 2 Study schedule of enrolment, interventions and assessments. $-\mathrm{T} 1=-2$ weeks, $0=$ baseline, $\mathrm{T} 1=$ week 1 , $\mathrm{T} 2=$ end of treatment, $\mathrm{T} 3=3$-month follow-up, $\mathrm{T} 4=6$-month follow-up and $T 5=12$-month follow-up.

\section{Blinding}

The nature of the intervention does not permit blinding participants or therapists to group assignment. Nonetheless, to minimise bias, before the intervention begins, all participants will be told that their assigned intervention aims to improve participation, and they will be asked to set personalised goals at the beginning of the intervention. Outcome assessors, data managers, statistical analysts, and principal and co-principal investigators are all blinded to group assignment. The allocation result (intervention or control group) are replaced with the Roman numerals 'I' or 'II' in the blind code. After the statistical analyses are completed, the grouping code will be revealed.

\section{Interventions}

\section{Experimental intervention group}

The OPASS programme will be delivered to the experimental intervention group. The programme was developed on the basis of the strategy training protocol described by Skidmore $e t a l^{27}$ and was refined considering experiences from a feasibility study. ${ }^{28}$ Participants will be engaged in the intervention in addition to their routine 


\section{Self-selected goals}

\section{Self-evaluation}

\section{Global strategy training Goal-Plan-Do-Check}

\section{Generalisation and Transfer}

Figure 3 Global strategy intervention procedure.

outpatient rehabilitation. Trained research therapists will deliver the intervention to participants in a quiet room at our recruitment sites.

The therapist and the participant will collaborate to identify three to five goals related to community participation that the participant perceives as important using the Canadian Occupational Performance Measure ${ }^{30}$ and Activity Card Sort. ${ }^{31}$ Next, the therapist will ask the participant to identify barriers to the performance of each activity. Using a global strategy (Goal-Plan-Do-Check) ${ }^{32}$ and expert facilitation (guided discovery), ${ }^{33}$ the therapist and the participant will work collaboratively to (1) set a goal to address the barriers, (2) develop a plan to address the goal, (3) implement the plan and (4) evaluate whether the plan worked or requires revision. During this process, the therapist will use a problem-solving framework to help the participant explore options to address the barriers of participation, plan actions, perform the plan, evaluate performance and generalise the learned skills. ${ }^{34}$ The procedure will be repeated iteratively until the participant goal is met or the participant decides to address the next goal.

Each intervention session will last $45 \mathrm{~min}$ and will be delivered twice weekly for a total of 12 to 15 sessions. At the end of each session, the therapist will prompt the participant to identify the principles learnt during the session and will encourage the participant to apply these principles in the next session (see figure 3). ${ }^{27}$

Caregivers or family members of participants will be invited to join each intervention session. The therapist will have a thorough discussion with the caregiver regarding the intervention and encourage the caregiver to provide support and supervision to the participant, as required, throughout the intervention. Caregiver involvement will be recorded for analysis.

\section{Control intervention group}

Participants in the control group will receive a dosematched attention control provided by trained therapists that control for attention from a skilled therapist and discussions about rehabilitation experiences. To be consistent with the intervention group, the control therapist and participant will first collaborate to identify three to five goals related to community participation that the participant perceives as important. Then, the therapist will use scripted questions to elicit participants to describe their experiences and their feelings regarding their condition and their usual care rehabilitation activities. The therapists will ask participants to document their feelings and experiences using journals between sessions and discuss their journal entries while the therapists use reflective listening skills. Each control intervention session will last $45 \mathrm{~min}$ and will be delivered twice weekly for a total of 12-15 sessions. Caregivers will also be invited to accompany participants to each session and their attendance will be recorded.

\section{Withdrawal or dropout criteria}

Participation in the trial will be terminated in case of the following: (1) being unwilling or unable to continue the trial, (2) exhibiting illness deterioration or any other physical or mental conditions that may impede their continued participation or (3) experiencing serious adverse events (AEs).

\section{Participant adherence and retention}

Patients' adherence in the intervention will be evaluated based on the attendance rate of the intervention sessions, engagement in intervention sessions (measured using the Pittsburgh Rehabilitation Participation Scale, ${ }^{35}$ a 6-point scale assessing effort and motivation of participating in the intervention session), and intervention adherence (measured using the Strategy Training Fidelity Checklist $^{36}$ developed by Skidmore et al) to evaluate to what degree the intervention has been delivered in line with what was planned. $20 \%$ of sessions will be randomly selected, for which two trained independent raters will fill out a checklist to assess therapists' (1) adherence to the principles in the intervention protocol (yes or no) and (2) competence in execution (inadequate, adequate or exceptional).

Several strategies will be used to enhance the adherence and retention of the participants. First, our research staff will provide a thorough introduction into the study to participants and their caregivers before they consent to participate. Second, during the intervention, the research therapists will develop a relationship with participants, encourage their continued participation in the study and supervise their adherence to the study protocol. Third, our research staff will maintain monthly telephone contact with participants throughout the study period to maintain the relationship. Finally, incentives will be provided to participants who complete all assessments and intervention sessions to encourage their active participation. 


\section{Outcome assessment}

Primary outcome

The primary outcome of this study is change in participation performance, as measured using the PM-3D4D, which is a 24-item measure designed to assess three domains of participation (productivity, social and community) ${ }^{3738}$ For each domain, respondents are asked to rate the items on the following four dimensions: (1) diversity, (2) frequency, (3) desire for change and (4) perceived difficulty. ${ }^{37}$ For the purpose of this study, the frequency and perceived difficulty dimensions will be adopted to reflect the objective and subjective participation performance of participants, respectively. The reliability, validity and responsiveness of the PM-3D4D have been examined in a stroke population in Taiwan. ${ }^{37-40}$

\section{Secondary outcomes}

The secondary outcomes of this study are changes in activity performance, cognitive function and self-efficacy.

Activity performance will be evaluated using the Activity Measure for Post-Acute Care (AM-PAC) generic outpatient short forms. The AM-PAC assesses the difficulty in performing three activity domains, namely basic mobility (18 items), daily activity (15 items) and applied cognitive (19 items) on a 4-point scale. A summary score for each subscale is transformed into standardised scores on a t-score scale. ${ }^{41}$ The Chinese version of the AM-PAC demonstrated strong psychometric properties in patients undergoing rehabilitation in Taiwan. ${ }^{42}$

Participants' general cognitive function will be assessed using the Montreal Cognitive Assessment, ${ }^{43}$ a 30-item assessment tool, covering visuospatial/executive, naming, memory, attention, language, abstraction, delayed recall and orientation. The scale exhibited promising reliability and higher sensitivity than that of the mini-mental state examination in screening for cognitive deficits in people who had a stroke. ${ }^{43}$ Executive function will be evaluated using the Stroop Test and the Trail Making Test (TMT A and B). The Stroop Test measures inhibition, set-shifting and selective attention. ${ }^{45}$ Participants will be asked to name the ink colour of a word as accurately and as rapidly as possible. The ink colour of the word could be congruent or incongruent with the written colour name. The time taken by each participant to complete the task will be calculated and recorded. ${ }^{46} 47$ The validity of the Stroop Test has been validated in patients with traumatic brain injury. ${ }^{48}$ The TMT A and B will be used to measure sustained attention, sequencing, mental flexibility and visual tracking. TMT A requires the individual to link a series of 25 numbered circles distributed randomly on a test paper as quickly as possible. ${ }^{49}$ TMT B requires the individual to link switch alternatively between a set of numbers (1-13) and a set of letters (A-L). The time taken to complete TMT A and B will be calculated; a longer completion time indicates a poorer outcome. This instrument was validated in diverse populations. ${ }^{50-52}$

The General Self-Efficacy Scale (GSES) ${ }^{53}$ is a 10 -item measure used to evaluate participants' perceived self-efficacy on a 4-point scale (from 1 'not at all true' to 4 'exactly true'). The summary score of the GSES ranges from 10 to 40, with a higher score indicating higher selfefficacy. ${ }^{53}$ The reliability of the GSES was validated in Chinese-speaking populations. ${ }^{54}$ All assessments will be administered by our research staff who are trained for administering these standardised measurement tools.

\section{Additional data collection}

At baseline, demographic characteristics of participants will be collected through a questionnaire developed by the research team. Clinical variables will be retrieved from medical charts. The National Institutes of Health Stroke Scale $^{55}$ will be used to characterise stroke severity. The modified Rankin Scale ${ }^{56}$ will be used to characterise the participant's disability level. The EuroQol-5-Dimension ${ }^{57}$ will be used to assess participants' health-related quality of life. The Healing Encounters and Attitudes Lists (HEAL) Positive Outlook questionnaire ${ }^{58}$ will be used to assess participants' positive attitude.

\section{Qualitative data}

Qualitative data will be collected through structured interviews with patients, their caregivers and the research therapists to understand their experience and satisfaction with the intervention and perceived effectiveness. Interviews will be conducted by well-trained interviewers at the end of each participant's intervention programme. All interviews will be audio-recorded and transcribed verbatim. In addition, field notes and research team meeting notes will be thoroughly documented.

\section{Safety evaluation}

Any unexpected, AEs that occur during the study period will be documented and reported to the study team to evaluate whether they are caused by the study. Serious AEs will be reported to the research ethics committee.

\section{Sample size}

For the primary research aim, the standard response means (SRMs) for the overall frequency and difficulty scales of the PM-3D4D from baseline to the 3-month follow-up were 0.67 and 1.05 , respectively. ${ }^{59}$ In the twogroup t-test, different SRMs between T1 (pre-intervention) and T2 (post-intervention) in the intervention group are expected, whereas no changes are expected in the control group. With a sample size of 84 for each group, $80 \%$ power will be obtained to detect an SRM as low as 0.44 . With a lost-to-follow-up rate of $20 \%$, a sample size of 105 is required for each group.

For the second research aim, a multiple regression model with a sample size of 210 (105 in each group), as estimated from the primary aim, will have $80 \%$ power to detect correlations of 0.2 or above between the score change in the secondary outcomes, with $10 \%$ of the variance explained by other background characteristics.

For the third aim, a simulation study will be conducted to calculate the power of the longitudinal study design. From a previous study that examined the responsiveness 


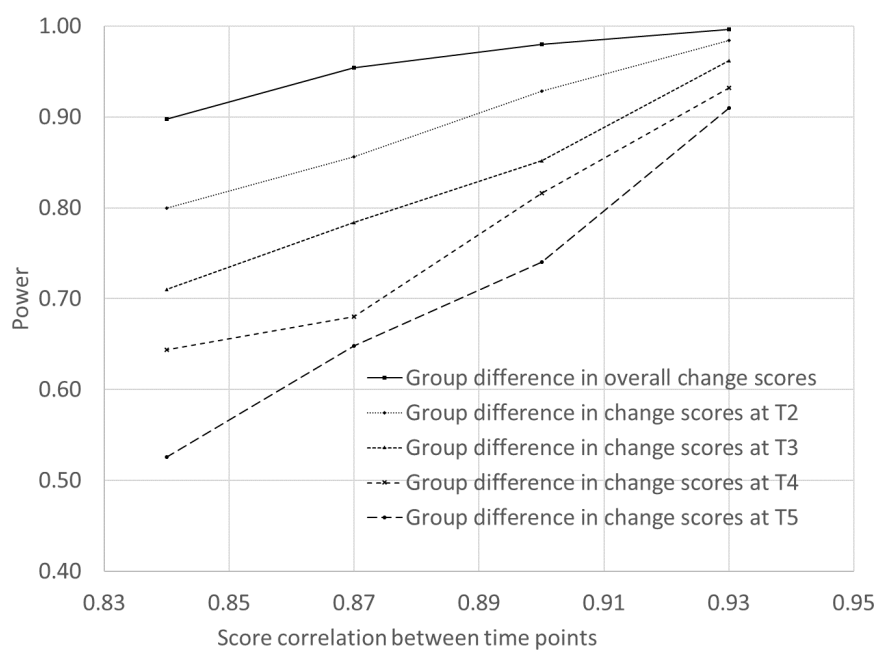

Figure 4 Estimation of research power through score correlation between time point.

of the PM-3D4D scale ${ }^{59}$ the effect size estimates $(0.23)$ will be adopted as the effect size in the intervention group for different follow-up times, with an assumption of no change in the control group. Four score correlations across time points $(0.84,0.87,0.90$ and 0.93$)$ and a lost-to-follow-up rate of $20 \%$ are expected. As illustrated in figure 4 , the power increases with the score correlation. With a sample size of 105 in each group and a score correlation over $0.9,80 \%$ power will be obtained to detect an effect size of 0.23 at time points $\mathrm{T} 1, \mathrm{~T} 2$ and $\mathrm{T} 3$, and $74 \%$ power to detect the same effect at $\mathrm{T} 4$.

\section{Statistical analysis}

Quantitative data analysis will be conducted using SAS V.9.3 (SAS institute). All analyses will be conducted on an intention-to-treat basis, where all participants are analysed in their assigned group regardless of their compliance with the intervention. Missing data will be imputed using a multiple imputation method. Vigorous attempts will be made to obtain follow-up assessments for all participants enrolled in the trial, regardless of their compliance with the treatment protocols. Sensitivity analysis will be conducted to explain differences in samples of complete and incomplete data.

Descriptive analysis will be conducted to describe the frequency, percentage distribution, mean and SD of all variables. Differences in demographic characteristics and baseline values between the treatment and control groups will be analysed using the $\chi^{2}$ test, the Mann-Whitney $\mathrm{U}$ test or the t-test, depending on the type of assessment scale (nominal, ordinal or interval) and normal distribution.

For our primary aim, determining the effectiveness of the OPASS programme to improve participation compared with that of the control protocol, the change in PM-3D4D scores from T1 to T2 between the intervention group and the control group will be compared by using a two-group t-test. Multiple linear regression models (equivalent to analysis of covariance) will be applied to examine whether the change in scores differs between groups after controlling for background characteristics.

For our second aim, to determine whether the changes in participation after the intervention are correlated with changes in applied cognition, daily activities, executive function and self-efficacy, the score change of these secondary outcome variables from $\mathrm{T} 1$ to $\mathrm{T} 2$ will be calculated. Multiple linear regression models will be used to examine the association between the score change in each of these secondary outcome variables and score changes in the PM-3D4D from T1 to T2. In the models, the interaction between the group variable and the score change in the secondary outcome variables will be included to assess whether the associations differ between the intervention and control groups.

To examine the intervention effect at different time points, constrained longitudinal data analysis (cLDA) will be applied to model the PM-3D4D, AM-PAC, Stroop Test, TMT A and B, and GSES scores at T1 (pre-intervention), T2 (post-intervention), T3 (3-month follow-up), T4 (6-month follow-up) and T5 (12-month follow-up). In the cLDA, both the baseline and the follow-up outcome variables will be treated as dependent variables. The model will include fixed effects of time, group variables, difference between study groups at each time point and background characteristics. The unstructured variancecovariance matrix will be used to account for repeated measures within each subject. The mean difference between the intervention and control groups for the score change at each time point and the corresponding $\mathrm{p}$ value will be reported. The significance will be set at $\mathrm{p}<0.05$.

Qualitative data will be analysed using thematic analysis, which involves a combination of inductive and deductive coding. ${ }^{60}$ Transcribed data will be coded by two independent coders and another researcher will review their coding results to ensure quality and consistency.

\section{Data management and monitoring}

The principal investigator is responsible for data storage and protection. All study documents will be stored securely and identified by a unique study identification number, which will be linked to the subject identifiers through a master code. Only the principal investigator and a restricted number of research staff will have access to the master code, which will be maintained in an encrypted file separately from the study data. All data will be entered into the dataset by research assistants using a unique identification number and will be stored in a secure password-protected drive. Two research assistants will be responsible for checking and auditing the data. All computers and electronic systems are in locked offices and all data can only be accessed by Institutional Review Board-approved study team members. Because of the minimal risks expected from this study and because no new drugs, biologics or devices are involved, a data monitoring committee is not required for this study. The Office of Human Research of Taipei Medical University will be 
in charge of monitoring and auditing data collected for this study.

All data will be archived for 10 years after study completion. Publications or presentations regarding the data obtained in this project will not include information that could identify those participating in this project.

\section{Patient and public involvement}

This trial is currently in the recruitment phase. No patients and/or public has been involved in the trial.

\section{Ethics and dissemination}

This study protocol conformed to the Declaration of Helsinki. Ethics approval of this study protocol and consent forms were obtained from the Taipei Medical University Institutional Review Board (approval number: N201804055). The study background, main objective and potential benefits and risks will be explained to participants before they sign informed consent forms. The study protocol has been registered and is available on the Clinical Trials Registry website. Major protocol modifications will require the approval of the ethics committee. Approved changes will be reported on the Clinical Trials Registry website. The study results will be provided to participants and be disseminated to researchers, healthcare providers and people with disabilities through presentations at scientific conferences, professional websites and publications in peer-reviewed journals.

\section{DISCUSSION}

To the best of our knowledge, this is the first study to evaluate the efficacy of strategy training for improving participation among stroke survivors with cognitive impairment in an Asian country. Both quantitative and qualitative data will be collected with multiple measurement tools and at different time points. A rigorous study design will be employed to control for potential bias, and the fidelity of the study will be closely monitored. The study results could help advance stroke rehabilitation practice and benefit stroke survivors in terms of their health, independence and transition to the community.

Although the strengths of this study are considerable, potential limitations must be acknowledged. First, this is a single-blind RCT. Similar to other non-pharmacological studies, our participants and interventionists cannot be blinded. Nevertheless, outcome assessors and data analysts will be blinded to avoid investigator bias. Second, considering the longitudinal nature of this study, considerable challenges in participant recruitment and retention may occur. Stroke survivors may refuse to participate in or drop out of our study because of their illness, family, transportation difficulties and other reasons. To address these challenges, our research team will collaborate with clinical staff at participating hospitals to recruit and retain participants. Additionally, we will develop rapport with our participants and their family by making phone calls on a regular basis throughout their participation in the study. Incentives will also be provided to participants who complete all assessments and intervention sessions to encourage their continued participation in the study. Finally, medical record was used to screen for depressive disorders. Without more sensitive screening tools, minor depressive symptoms, which may be associated with cognitive functions, may be overlooked. Future studies will need to include more sensitive tools to assess minor depressive symptoms.

This study will determine whether this newly developed intervention programme significantly improves shortterm and long-term participation outcomes among stroke survivors with cognitive impairment. If our hypotheses are confirmed, this intervention programme could not only be applied in rehabilitation practice and benefit patients during their transition from hospital to community but also considerably reduce the long-term care burden on family and society.

\section{Author affiliations}

${ }^{1}$ Graduate Institute of Injury Prevention and Control, College of Public Health, Taipei Medical University, Taipei, Taiwan

${ }^{2}$ Department of Physical Medicine and Rehabilitation, School of Medicine, College of Medicine, Taipei Medical University, Taipei, Taiwan

${ }^{3}$ Department of Physical Medicine and Rehabilitation, Taipei Tzu Chi Hospital, Taipei, Taiwan

${ }^{4}$ Health Law, Policy \& Management; Biostatistics and Epidemiology Data Analytics Center, Boston University School of Public Health, Boston, Massachusetts, USA

${ }^{5}$ Department of Physical Medicine and Rehabilitation, Taipei Municipal Wan-Fang Hospital, Taipei, Taiwan

${ }^{6}$ Department of Physical Medicine and Rehabilitation, Taipei Medical University Hospital, Taipei, Taiwan

${ }^{7}$ Department of Physical Medicine and Rehabilitation, Shuang Ho Hospital, Taipei, Taiwan

${ }^{8}$ Department of Physical Medicine and Rehabilitation, National Taiwan University Hospital, Taipei, Taiwan

${ }^{9}$ Department of Physical Medicine and Rehabilitation, College of Medicine, National Taiwan University, Taipei, Taiwan

${ }^{10}$ Department of Physical Medicine and Rehabilitation, National Taiwan University Hospital Beihu Branch, Taipei, Taiwan

${ }^{11}$ Department of Occupational Therapy, School of Health and Rehabilitation Sciences, University of Pittsburgh, Pittsburgh, Pennsylvania, USA

${ }^{12}$ Department of Physical Medicine \& Rehabilitation, School of Medicine, University of Pittsburgh, Pittsburgh, Pennsylvania, USA

Acknowledgements We thank our collaborating therapists, including Yi-Hsuan Wu, OTR/L (National Taiwan University Hospital), Jui-Chi Lin, OTR/L (Shuang Ho Hospital), Hsien-Lin Cheng, OTR/L (Taipei Medical University Hospital), Wan-Chi Lin, OTR/L (Wan Fang Hospital), Cheng-Hsun Yang, OTR/L (Wan Fang Hospital), Han-Ting Tsai, OTR/L (Wan Fang Hospital), Yen-Ting Liu, OTR/L (Wan Fang Hospital), Chi-Tzu Feng, OTR/L (Taipei Tzu Chi Hospital) and Yi-Chun Lu, OTR/L (Taipei Tzu Chi Hospital).

Collaborators Yi-Hsuan Wu; Jui-Chi Lin; Hsien-Lin Cheng; Wan-Chi Lin; ChengHsun Yang; Han-Ting Tsai; Yen-Ting Liu; Chi-Tzu Feng; and Yi-Chun Lu.

Contributors FHC led the grant application. FHC, YNL, JHK, THL, LL and ERS contributed to the study protocol and conceived, designed and obtained funding for the study. FHC, VC and PN led the writing of the manuscript. PN led the statistical design and sample size estimation. VC, YNL, JHK, THL, LL and DSH contributed to the implementation and coordination of the study. ERS made substantial contributions to the study design, writing and critical review of the intellectual content of the manuscript. All authors contributed to revising the manuscript and have read and approved the final version.

Funding This work is supported by the National Health Research Institutes, Taiwan (grant number NHRI-EX108-10819PC), the Ministry of Science and Technology, Taiwan (grant number MOST108-2314-B-038-030-MY3) and in part by the 
Ministry of Education, Taiwan (grant numbers DP2-108-21121-01-A-01-01 and DP2-109-21121-01-N-09-01).

Competing interests None declared.

Patient consent for publication Not required.

Provenance and peer review Not commissioned; externally peer-reviewed.

Supplemental material This content has been supplied by the author(s). It has not been vetted by BMJ Publishing Group Limited (BMJ) and may not have been peer-reviewed. Any opinions or recommendations discussed are solely those of the author(s) and are not endorsed by BMJ. BMJ disclaims all liability and responsibility arising from any reliance placed on the content. Where the content includes any translated material, BMJ does not warrant the accuracy and reliability of the translations (including but not limited to local regulations, clinical guidelines, terminology, drug names and drug dosages), and is not responsible for any error and/or omissions arising from translation and adaptation or otherwise.

Open access This is an open access article distributed in accordance with the Creative Commons Attribution Non Commercial (CC BY-NC 4.0) license, which permits others to distribute, remix, adapt, build upon this work non-commercially, and license their derivative works on different terms, provided the original work is properly cited, appropriate credit is given, any changes made indicated, and the use is non-commercial. See: http://creativecommons.org/licenses/by-nc/4.0/.

\section{ORCID iD}

Feng-Hang Chang http://orcid.org/0000-0002-0711-3644

\section{REFERENCES}

1 Feigin VL, Norrving B, Mensah GA. Global burden of stroke. Circ Res 2017;120:439-48.

2 World Health Organization. The atlas of heart disease and stroke 2015. Available: http://www.who.int/cardiovascular_diseases/ resources/atlas/en/ [Accessed 23 September 2015].

3 Chen C-C, Chen C-S, Liu T-C, et al. Stock or stroke? stock market movement and stroke incidence in Taiwan. Soc Sci Med 2012;75:1974-80.

4 Lin H-C, Lin Y-J, Liu T-C, et al. Urbanization and stroke prevalence in Taiwan: analysis of a nationwide survey. J Urban Health 2007;84:604-14.

5 Adamit T, Maeir A, Ben Assayag E, et al. Impact of first-ever mild stroke on participation at 3 and 6 month post-event: the TABASCO study. Disabil Rehabil 2015;37:667-73.

6 Sun J-H, Tan L, Yu J-T. Post-Stroke cognitive impairment: epidemiology, mechanisms and management. Ann Trans/ Med 2014;2:80.

7 Cumming TB, Marshall RS, Lazar RM. Stroke, cognitive deficits, and rehabilitation: still an incomplete picture. Int J Stroke 2013;8:38-45.

8 Flynn RWV, MacWalter RSM, Doney ASF. The cost of cerebral ischaemia. Neuropharmacology 2008;55:250-6.

9 Ma VY, Chan L, Carruthers KJ. Incidence, prevalence, costs, and impact on disability of common conditions requiring rehabilitation in the United States: stroke, spinal cord injury, traumatic brain injury, multiple sclerosis, osteoarthritis, rheumatoid arthritis, limb loss, and back pain. Arch Phys Med Rehabil 2014;95:986-95.

10 Wade DT, de Jong BA. Recent advances in rehabilitation. BMJ 2000;320:1385-8.

11 World Health Organization. International classification of functioning, disability and health (ICF. Geneva: World Health Organization, 2001.

12 Chang F-H, Coster WJ. Conceptualizing the construct of participation in adults with disabilities. Arch Phys Med Rehabil 2014:95:1791-8.

13 Lai S-M, Studenski S, Duncan PW, et al. Persisting consequences of stroke measured by the stroke impact scale. Stroke 2002;33:1840-4

14 Woodman P, Riazi A, Pereira C, et al. Social participation post stroke: a meta-ethnographic review of the experiences and views of community-dwelling stroke survivors. Disabil Rehabil 2014;36:2031-43.

15 Ahern MM, Hendryx M. Community participation and the emergence of late-life depressive symptoms: differences between women and men. J Womens Health 2008;17:1463-70.

16 Yu B, Steptoe A, Niu K, et al. Prospective associations of social isolation and loneliness with poor sleep quality in older adults. Qual Life Res 2018;27:683-91.

17 Xia N, Li H. Loneliness, social isolation, and cardiovascular health. Antioxid Redox Signal 2018;28:837-51.

$18 \mathrm{Kim} \mathrm{M}$, Cho K, Lee W. Community walking training program improves walking function and social participation in chronic stroke patients. Tohoku J Exp Med 2014;234:281-6.
19 Barclay RE, Stevenson TJ, Poluha W, et al. Interventions for improving community ambulation in individuals with stroke. Cochrane Database Syst Rev 2015;94:CD010200.

20 Mayo NE, Anderson S, Barclay R, et al. Getting on with the rest of your life following stroke: a randomized trial of a complex intervention aimed at enhancing life participation post stroke. Clin Rehabil 2015;29:1198-211.

21 Kumar KS, Samuelkamaleshkumar S, Viswanathan A, et al. Cognitive rehabilitation for adults with traumatic brain injury to improve occupational outcomes. Cochrane Database Syst Rev 2017;6:CD007935.

22 Skidmore ER, Holm MB, Whyte EM, et al. A case report examining the feasibility of Meta-Cognitive strategy training in acute inpatient stroke rehabilitation. Neuropsychol Rehabil 2011;21:208-23.

23 Skidmore ER, Butters M, Whyte E, et al. Guided training relative to direct skill training for individuals with cognitive impairments after stroke: a pilot randomized trial. Arch Phys Med Rehabil 2017;98:673-80.

24 Dawson DR, McEwen S, Polatajko H. Enabling participation across the lifespan: advancements, adaptations and extensions of the CO-OP approach. Bethesda, MD: American Occupational Therapy Association, 2017.

25 Novakovic-Agopian T, Chen AJ-W, Rome S, et al. Rehabilitation of executive functioning with training in attention regulation applied to individually defined goals: a pilot study bridging theory, assessment, and treatment. $J$ Head Trauma Rehabil 2011;26:325-38.

26 Levine B, Schweizer TA, O'Connor C, et al. Rehabilitation of executive functioning in patients with frontal lobe brain damage with goal management training. Front Hum Neurosci 2011;5:9.

27 Skidmore ER, Dawson DR, Butters MA, et al. Strategy training shows promise for addressing disability in the first 6 months after stroke. Neurorehabil Neural Repair 2015;29:668-76.

28 Lin Y-N, Wu C-Y, Yeh P-C, et al. Adapting strategy training for adults with acquired brain injury: a feasibility study in a Chinese population. Am J Occup Ther 2020;74:7403205130p1.

29 Larson EB, Heinemann AW. Rasch analysis of the executive interview (the EXIT-25) and introduction of an abridged version (the quick exit). Arch Phys Med Rehabil 2010;91:389-94.

30 Law M, Baptiste S, Carswell A, et al. Canadian occupational performance measure manual. Toronto, ON: CAOT Publications ACE, 2005.

31 Baum CM, Edwards D. Activity Card Sort (ACS). Bethesda, MD: American Occupational Therapy Association, Inc, 2008.

32 Meichenbaum D. Cognitive-behavior modification: an integrative approach. New York: Plenum Press, 1977.

33 Wales CE, Nardi AH, Stager R A. Decision making: new paradigm for education. Educational Leadership 1986;43:203-15.

34 Graham F, Rodger S, Ziviani J. Enabling occupational performance of children through coaching parents: three case reports. Phys Occup Ther Pediatr 2010;30:4-15.

35 Lenze EJ, Munin MC, Quear T, et al. The Pittsburgh rehabilitation participation scale: reliability and validity of a clinician-rated measure of participation in acute rehabilitation. Arch Phys Med Rehabil 2004;85:380-4.

36 Skidmore ER, Dawson DR, Whyte EM, et al. Developing complex interventions: lessons learned from a pilot study examining strategy training in acute stroke rehabilitation. Clin Rehabil 2014;28:378-87.

37 Chang F-H, Liou T-H, Ni P, et al. Development of the participation Measure-3 domains, 4 dimensions (PM-3D4D): a new outcome measure for rehabilitation. Arch Phys Med Rehabil 2017;98:286-94.

38 Chang F-H, Ni P. Responsiveness and predictive validity of the participation Measure-3 domains, 4 dimensions in survivors of stroke. Arch Phys Med Rehabil 2019;100:2283-92

39 Chang $\mathrm{F}-\mathrm{H}$. Measuring participation of rehabilitation patients: testretest reliability and mode of administration concordance of the participation Measure-3 domains, 4 dimensions (PM-3D4D). Arch Phys Med Rehabil 2017;98:1622-7.

40 Chang F-H, Chang K-H, Liou T-H, et al. Validation of the participation Measure-3 domains, 4 dimensions (PM-3D4D). Arch Phys Med Rehabil 2017;98:2498-506.

41 Jette AM, Haley SM, Ni P, et al. Adaptive short forms for outpatient rehabilitation outcome assessment. Am J Phys Med Rehabil 2008;87:842-52

42 Chang F-H, Liou T-H, Brodersen J, et al. Adaptation of the activity measure post-acute care (AM-PAC) from English to mandarin using the dual-panel translation approach. Disabil Rehabil 2018;40:2571-6.

43 Dong Y, Sharma VK, Chan BP-L, et al. The Montreal cognitive assessment (MoCA) is superior to the Mini-Mental state examination (MMSE) for the detection of vascular cognitive impairment after acute stroke. J Neurol Sci 2010;299:15-18. 
44 Nasreddine ZS, Phillips NA, Bédirian V, et al. The Montreal cognitive assessment, MoCA: a brief screening tool for mild cognitive impairment. J Am Geriatr Soc 2005;53:695-9.

45 Stroop JR, Ridley Stroop J. Studies of interference in serial verbal reactions. J Exp Psychol 1992;121:15-23.

46 Koss E, Ober BA, Delis DC, et al. The Stroop color-word test: indicator of dementia severity. Int J Neurosci 1984;24:53-61.

47 Ridley DR, Johnson DE, Braisted PD. The color-word connotative incongruity effect. Percept Mot Skills 1978;46:939-46.

48 Ponsford J, Kinsella G. Attentional deficits following closed-head injury. J Clin Exp Neuropsychol 1992;14:822-38.

49 Bowie CR, Harvey PD. Administration and interpretation of the TRAIL making test. Nat Protoc 2006;1:2277-81.

50 Shum DHK, McFarland KA, Bain JD. Construct validity of eight tests of attention: comparison of normal and closed head injured samples. Clinical Neuropsychologist 1990;4:151-62.

51 Lezak MD, Howieson DB, Bigler ED, et al. Neuropsychological Assessment. 5th ed. Oxford, NY: Oxford University Press, 2012.

52 Zakzanis KK, Mraz R, Graham SJ. An fMRI study of the TRAIL making test. Neuropsychologia 2005;43:1878-86.

53 Schwarzer R, Jerusalem M. Generalized self-efficacy scale. Measures in health psychology: a user's portfolio causal and control beliefs. Windsor, UK: NFER-NELSON, 1995: 35-7.
54 Liu L, Xu N, Wang L. Moderating role of self-efficacy on the associations of social support with depressive and anxiety symptoms in Chinese patients with rheumatoid arthritis. Neuropsychiatr Dis Treat 2017;13:2141-50.

55 Cincura C, Pontes-Neto OM, Neville IS, et al. Validation of the National Institutes of health stroke scale, modified Rankin scale and Barthel index in Brazil: the role of cultural adaptation and structured interviewing. Cerebrovasc Dis 2009;27:119-22.

56 Patel N, Rao VA, Heilman-Espinoza ER, et al. Simple and reliable determination of the modified Rankin scale score in neurosurgical and neurological patients: the mRS-9Q. Neurosurgery 2012;71:971-5.

57 Tsuchiya A, Ikeda S, Ikegami N, et al. Estimating an EQ$5 \mathrm{D}$ population value set: the case of Japan. Health Econ 2002;11:341-53.

58 Greco CM, Yu L, Johnston KL, et al. Measuring nonspecific factors in treatment: item banks that assess the healthcare experience and attitudes from the patient's perspective. Qual Life Res 2016;25:1625-34.

59 Chang F-H, Ni P. Responsiveness and predictive validity of the participation Measure-3 domains, 4 dimensions in survivors of stroke. Arch Phys Med Rehabil 2019;100:2283-92.

60 Patton MQ. Qualitative Research \& Evaluation Methods. Thousand Oaks, CA: Sage Publications, Inc, 2002. 